\title{
Evaluation of a new quantitative test for HCV core Ag
}

Anna Rodella', Luigina Terlenghi', Francesco Gargiulo', Claudio Galli², Nino Manca'

I Department of Microbiology and Virology, University of Brescia

2 Abbott Diagnostics, Roma

Key Words: HCV, core Ag, CMIA

Valutazione di un nuovo test quantitative per $\mathrm{HCV}$ core $\mathbf{A g}$

\section{SUMMARY}

Introduction. The quantitative determination of viral antigens is a diagnostic innovation.

The availability of an automated test for detecting the "core" antigen of the hepatitis C virus (HCV) allowed to assess its characteristics and potential applications.

Methods. The Abbott ARCHITECT HCV core Ag assay is a fully automated CMIA measuring HCV core antigen at concentrations between 3 and $180,000 \mathrm{fmol} / \mathrm{L}$. The evaluation has been conducted on anti-HCV positive samples at various levels: on samples for which the quantitative (bDNA) and / or qualitative (TMA) HCV-RNA have been tested and on samples of hemodialysis patients.

Results. A positivity for HCV Ag was detectable in 10//42 (7.0\%) sera with single anti-core reactivity at additional RIBA 3 test while 58 samples reactive to NS3 or NS4 or NS5 were all HCV Ag negative. The frequency of Ag positivity was correlated with the anti-HCV signal (at anti-HCV ARCHITECT), ranging from $71 \%$ in samples with $\mathrm{S} / \mathrm{CO}>5$ to $6 \%$ in samples with $\mathrm{S} / \mathrm{CO}<5$. The antigen was detectable at low concentrations (average $50 \pm \mathrm{I} .17 \mathrm{fmol} / \mathrm{L}$ ) in $8 / 53$ samples (I5. I\%) with viremia below $500 \mathrm{IU} / \mathrm{mL}$. On samples with quantifiable HCV-RNA, the correlation between bDNA and HCV Ag was very good. Of the 65 examined hemodialysis patients, 46 were positive for both antibodies and antigen, 15 for only antibodies, 3 negative for both and one was highly positive for $\mathrm{HCV} \mathrm{Ag}$ (and HCV-RNA), but anti-HCV negative.

Conclusion. The sensitivity and the practicality of the new quantitative test for $\mathrm{HCV} A g$ allow to envisage the use for the evaluation of active $\mathrm{HCV}$ infection in anti-HCV positive patients, monitoring patients at risk of infection and as a complement of quantitative viremia.

\section{INTRODUZIONE}

La determinazione quantitativa di antigeni virali rappresenta un'innovazione diagnostica.

La disponibilità di un test automatizzato per la ricerca dell'antigene core del virus dell'epatite C (HCV) ha permesso di valutarne le caratteristiche e le potenziali applicazioni.

\section{MATERIALI E METODI}

Il test Abbott ARCHITECT HCV Core Ag è un dosaggio CMIA (Chemiluminescent Microparticle Immunoassay) completamente automatizzato che misura l'antigene core di HCV a concentrazioni comprese tra 3 e $180.000 \mathrm{fmol} / \mathrm{L}$. La valutazione è stata condotta su campioni positivi per antiHCV a diversi livelli: su 200 campioni con una singola reattività anticorpale al test RIBA 3 (Recombinant ImmunoBlot Assay), 115 campioni sui quali era stata eseguita la determinazione quantitativa (bDNA=branched Chain DNA) e/o qualitativa (TMA=Transcription Mediated Amplification) di HCV-RNA e su 65 campioni di pazienti in emodialisi.

\section{RISULTATI}

I dati di riproducibilità interdosaggio sui controlli accessori sono riportati in Tabella 1.

Una positività per HCV Ag era rilevabile in 10/142 (7.0\%) sieri con singola reattività per anti-core con il test supplementare RIBA 3 mentre i 58 campioni reattivi per NS3 o NS4 o NS5 erano tutti HCV Ag negativi (Tabella 2).

La frequenza di positività per l'antigene era correlata con il segnale anti-HCV (ARCHITECT), variando dal $71 \%$ in campioni con $\mathrm{S} / \mathrm{CO}>5$ al $6 \%$ nei campioni con $\mathrm{S} / \mathrm{CO}<5$ (Figura I).

L'antigene era rilevabile a basse concentrazioni (media $50 \pm 1.17 \mathrm{fmol} / \mathrm{L}$ ) in 8/53 campioni (15.1\%) con viremia inferiore a $500 \mathrm{UI} / \mathrm{mL}$. Sui campioni con HCV-RNA quantizzabile la correlazione tra bDNA e HCV Ag è risultata molto buona (Figura II).

Il confronto dei dati per HCV Ag e HCV-RNA in relazione ai vari genotipi dell' $\mathrm{HCV}$ è riportato in tabella 3 . Dei 65 pazienti in emodialisi esaminati, 46 (72.4\%) erano positivi sia per anticorpi che per antigene, 15 (21.5\%) solo per anticorpi, 3 (4.6\%) negativi per entrambi mentre 1
(1.5\%) era altamente positivo per HCV Ag (e per RNA) e anti-HCV negativo (Figura III). Il Profilo di monitoraggio per HCV Ag e HCV-RNA in un paziente in corso di terapia antivirale con interferone pegilato + ribavirina è riportato in Figura IV.

Tabella I. HCV Ag: riproducibilità interdosaggio sui controlli accessori.

\begin{tabular}{|c|c|c|c|}
\hline Prog. & $\begin{array}{c}\text { Architect } \\
\text { HCV Ag } \\
\text { neg }\end{array}$ & $\begin{array}{c}\text { Architect } \\
\text { HCV Ag } \\
\text { pos 1 }\end{array}$ & $\begin{array}{c}\text { Architect } \\
\text { HCV Ag } \\
\text { pos 2 }\end{array}$ \\
\hline 1 & 0,00 & 44,54 & 341,53 \\
\hline 2 & 0,87 & 45,16 & 286,07 \\
\hline 3 & 0,67 & 49,85 & 325,16 \\
\hline 4 & 0,54 & 47,79 & 284,85 \\
\hline 5 & 1,13 & 51,16 & 343,69 \\
\hline 6 & 0,00 & 44,55 & 283,92 \\
\hline 7 & 0,71 & 45,79 & 320,68 \\
\hline 8 & 0,00 & 44,27 & 310,10 \\
\hline 9 & 0,17 & 52,19 & 326,10 \\
\hline 10 & 0,61 & 41,05 & 289,81 \\
\hline 11 & 0,00 & 45,65 & 323,43 \\
\hline 12 & 0,00 & 44,16 & 301,39 \\
\hline
\end{tabular}

\begin{tabular}{|c|c|c|c|}
\hline Numero & 12 & 12 & 12 \\
\hline Media & 0,39 & 46,35 & 311,39 \\
\hline d.s. & 0,41 & 3,27 & 21,86 \\
\hline Mediana & 0,36 & 45,41 & 315,39 \\
\hline CV & n.a. & 7,05 & 7,02 \\
\hline Min & 0,00 & 41,05 & 283,92 \\
\hline Max & 1,13 & 52,19 & 343,69 \\
\hline
\end{tabular}


Tabella 2. HCV Ag in campioni monoreattivi anti-HCV.

\begin{tabular}{|c|c|c|c|}
\hline $\begin{array}{c}\text { Banda } \\
\text { RIBA 3 }\end{array}$ & N. & HCV Ag+ & $\%$ \\
\hline Core & 142 & 10 & $7,0 \%$ \\
\hline NS3 & 54 & 0 & $0,0 \%$ \\
\hline NS4 & 3 & 0 & $0,0 \%$ \\
\hline NS5 & 1 & 0 & $0,0 \%$ \\
\hline Totale & 200 & 10 & $5,0 \%$ \\
\hline
\end{tabular}

Tabella 3. HCV-RNA e HCV Ag per genotipo HCV.

\begin{tabular}{|c|c|c|c|c|}
\hline Genotipo & $\begin{array}{c}\text { N. } \\
\text { campioni }\end{array}$ & $\begin{array}{c}\text { HCV-RNA } \\
\text { positivi }\end{array}$ & $\begin{array}{c}\text { HCV Ag } \\
\text { positivi }\end{array}$ & $\%$ pos. \\
\hline $1 / 1 \mathrm{a}$ & 26 & 26 & 26 & 100 \\
\hline $1 \mathrm{~b}$ & 34 & 34 & 34 & 100 \\
\hline 2 & 3 & 3 & 3 & 100 \\
\hline $2 \mathrm{a} / 2 \mathrm{c}$ & 16 & 16 & 15 & 93,7 \\
\hline $3 \mathrm{a}$ & 24 & 24 & 22 & 91,7 \\
\hline $4 / 4 \mathrm{c} / 4 \mathrm{~d}$ & 12 & 12 & 11 & 90,9 \\
\hline
\end{tabular}

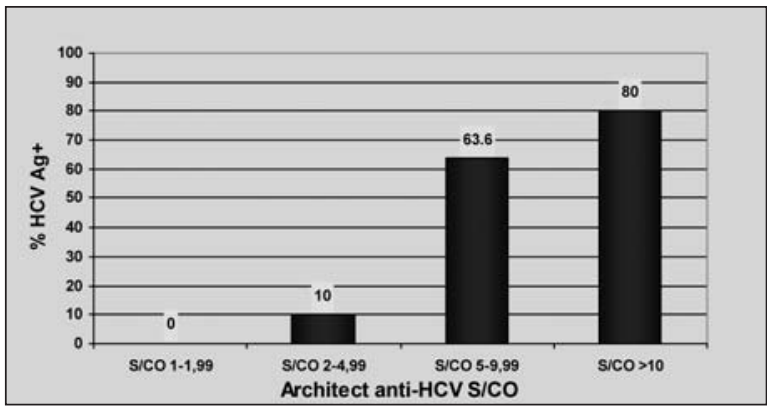

Figura I. Positività per HCV Ag in relazione con il livello di positività anticorpale.

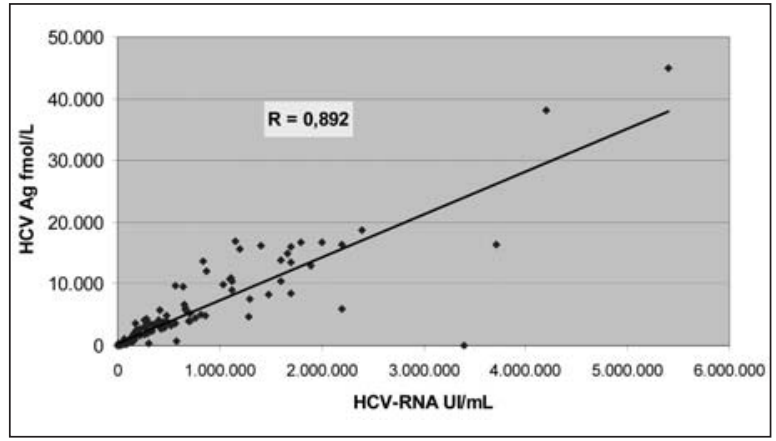

Figura 2. Correlazione tra HCV-RNA e HCV Ag.

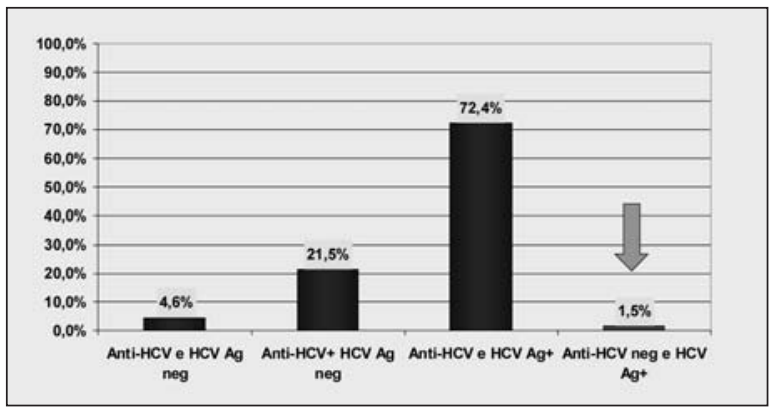

Figura III. Marcatori di infezione da HCV in 65 pazienti dializzati.

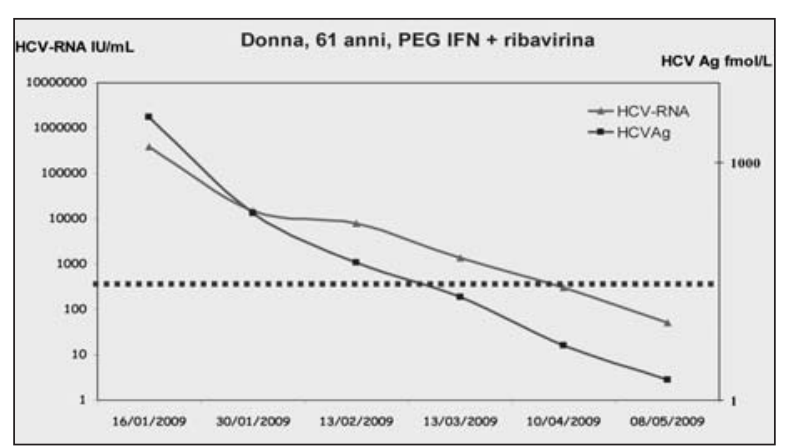

Figura IV. Profilo di monitoraggio in corso di terapia antivirale.

\section{CONCLUSIONI}

La sensibilità e la praticità di esecuzione del nuovo test quantitativo per HCV Ag consentono di ipotizzarne l'impiego per la valutazione delle infezioni attive da HCV in pazienti anti-HCV positivi, nel monitoraggio dei soggetti a rischio di infezione e come complemento della viremia quantitativa (1).

\section{BIBLIOGRAFIA}

1. Mederacke I, Wedemeyer H, Ciesek S, et al. Performance and clinical utility of a novel fully automated quantitative $\mathrm{HCV}$-core antigen assay. J Clin Virol, 2009; 46: 210-5. 